Article

\title{
Optimization of Preparation and Preclinical Pharmacokinetics of Celastrol-Encapsulated Silk Fibroin Nanoparticles in the Rat
}

\author{
Felicia Onyeabor ${ }^{1,+}$, Amy Paik ${ }^{1,+}$, Surya Kovvasu ${ }^{1, *}$, Baoyue Ding ${ }^{2}$, Jelissa Lin ${ }^{1}$, \\ Md Arif Wahid ${ }^{1}$, Sunil Prabhu ${ }^{1}$, Guru Betageri ${ }^{1}$ and Jeffrey Wang ${ }^{1, *(D)}$ \\ 1 Department of Pharmaceutical Sciences, College of Pharmacy, Western University of Health Sciences, \\ Pomona, CA 91766, USA \\ 2 Department of Pharmaceutics, College of Medicine, Jiaxing University, Jiaxing 314000, China \\ * Correspondence: skovvasu@westernu.edu (S.K.); jwang@westernu.edu (J.W.) \\ + These authors contributed equally to this work.
}

Received: 23 August 2019; Accepted: 4 September 2019; Published: 8 September 2019

check for updates

\begin{abstract}
Celastrol (CL), a bioactive compound isolated from Tripterygium wilfordii, has demonstrated bioactivities against a variety of diseases including cancer and obesity. However, its poor water solubility and rapid in vivo clearance limit its clinical applications. To overcome these limitations, nanotechnology has been employed to improve its pharmacokinetic properties. Nanoparticles made of biological materials offer minimal adverse effects while maintaining the efficacy of encapsulated therapeutics. Silk fibroin (SF) solution was prepared successfully by extraction from the cocoons of silkworms, and a final concentration of $2 \mathrm{mg} / \mathrm{mL} \mathrm{SF}$ solution was used for the preparation of CL-loaded SF nanoparticles (CL-SFNP) by the desolvation method. A stirring speed of $750 \mathrm{rpm}$ and storage time of $20 \mathrm{~h}$ at $-20^{\circ} \mathrm{C}$ resulted in optimized product yield. A high-performance liquid chromatography (HPLC) method was developed and validated for the analysis of CL in rat plasma in terms of selectivity, linearity, intra-/inter-day precision and accuracy, and recovery. No interference was observed in rat plasma. Linearity in the concentration range of $0.05-5 \mu \mathrm{g} / \mathrm{mL}$ was observed with $R^{2}$ of 0.999 . Precision and accuracy values were below the limit of acceptance criteria, i.e., $15 \%$ for quality control (QC) samples and 20\% for lower limit of quantification (LLOQ) samples. Rats were given intravenous (IV) administration of $1 \mathrm{mg} / \mathrm{kg}$ of pure CL in PEG 300 solution or CL-SFNP. The pharmacokinetic profile was improved with CL-SFNP compared to pure CL. Pure CL resulted in a maximum concentration $\left(\mathrm{C}_{\max }\right)$ value of $0.17 \mu \mathrm{g} \mathrm{mL} \mathrm{m}^{-1}$ at $5 \mathrm{~min}$ following administration, whereas that for CL-SFNP was $0.87 \mu \mathrm{g} \mathrm{mL}-1$ and the extrapolated initial concentrations $\left(\mathrm{C}_{0}\right)$ were 0.25 and $1.09 \mu \mathrm{g} \mathrm{mL} \mathrm{L}^{-1}$, respectively, for pure CL and CL-SFNP. A 2.4-fold increase in total area under the curve $\left(\mathrm{AUC}_{0 \text {-inf }}\right)\left(\mu \mathrm{g} \mathrm{h} \mathrm{mL} \mathrm{mL}^{-1}\right)$ was observed with CL-SFNP when compared with pure CL. CL-SFNP demonstrated longer mean residence time (MRT; $0.67 \mathrm{~h}$ ) than pure CL $(0.26 \mathrm{~h})$. In conclusion, the preparation of CL-SFNP was optimized and the formulation demonstrated improved pharmacokinetic properties compared to CL in solution following IV administration.
\end{abstract}

Keywords: silk fibroin nanoparticles; celastrol; optimized formulation; bioanalysis; pharmacokinetics

\section{Introduction}

Pancreatic ductal adenocarcinoma (PDAC) is the major form of malignancy in the pancreas [1]. Unsatisfactory results of conventional chemotherapeutic options demand the search for more effective anticancer agents. Recently, traditional Chinese medicines have received increasing attention in the scientific community for potential clinical applications [2]. 
The Chinese herb Tripterygium wilfordii (TW), also known as Lei Gong Teng (literally meaning thunder god vine) has demonstrated many bioactivities. The herbal extract as well as TW, two major bioactive compounds, triptolide (TP) and celastrol (CL, Figure 1), have attracted widespread interest in the research fields of Alzheimer's disease, rheumatoid arthritis, asthma, hypertension, and systemic lupus erythematosus [3]. Recently, several investigations revealed the potential use of CL in treating obesity $[4,5]$. Both CL and TP alone and in combination have demonstrated anticancer activity against a number of cancers [6,7]. The anti-proliferative effects of CL are centered on its ability to induce apoptosis in cancer cells derived from the pancreas, lung, glia, prostate, blood, and breast. In addition, $C L$ exhibits the ability to suppress angiogenesis and is a potent proteasome inhibitor [8,9]. However, the clinical use of CL is limited due to its poor solubility and unsatisfactory pharmacokinetic and side effect profiles $[8,10]$. Recently, biopolymer-based nanoparticles have attracted widespread attention for the use of biocompatible materials, which possess properties of low toxicity and biodegradability. Examples of biopolymer nanoparticles (NPs) include albumin, gelatin, and silk fibroin (SF) [11,12]. SF is a fibrous protein polymer in silk generated from the cocoon of domesticated silkworms, Bombyx mori. SF exhibits slow degradation, stealth mechanical properties, favorable processability, good biodegradability, and regenerative properties, which makes this biomaterial suitable in biomedicine and nanomedicine [12-16]. The protein structure of SF is comprised of a heavy chain $(\sim 325 \mathrm{kDa})$ and a light chain $(\sim 125 \mathrm{kDa})$. The secondary structure of SF consists of re-oriented $\beta$-sheets, which make up the hydrophobic block of SF. Hydrogen bonds in the $\beta$-sheet domain contribute to the strength and stability of the fiber in SF. The hydrophilic block is the amorphous domain of the SF structure, which contributes to the strength and stability of the fiber [12-16].

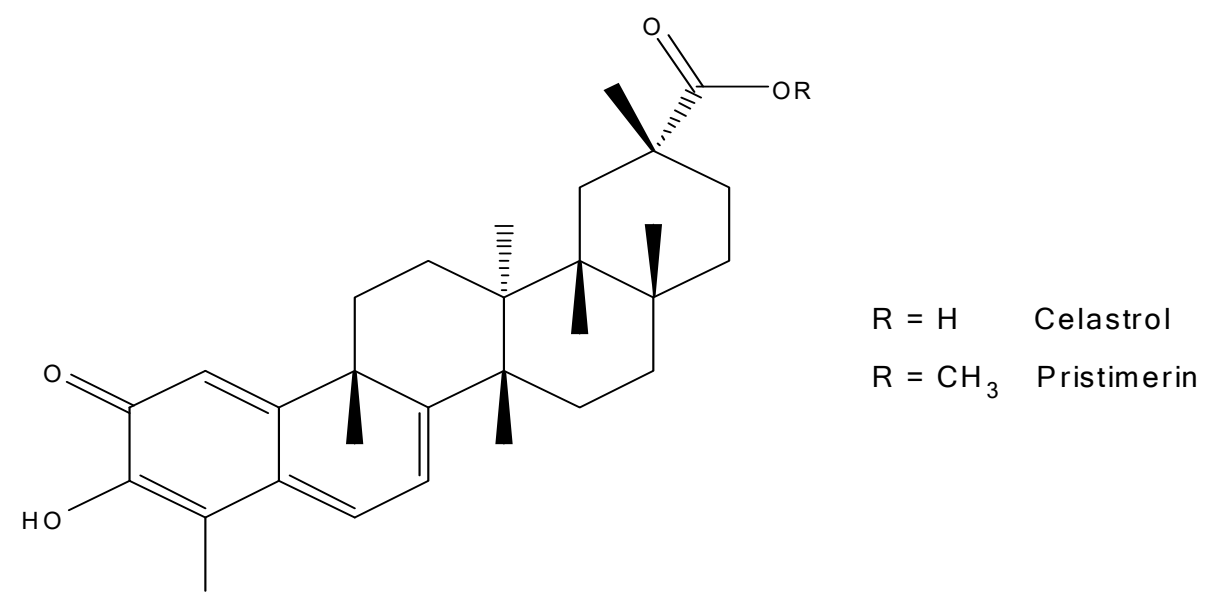

Figure 1. Chemical structures of celastrol (CL) and pristimerin.

We previously prepared CL-loaded SF nanoparticles (CL-SFNP) using a desolvation method [17]. In vitro drug release studies of CL-SFNP revealed a slow and sustained release of drug at physiological $\mathrm{pH}(\mathrm{pH}$ 7.4) and rapid release at lysosomal $\mathrm{pH}(\mathrm{pH}$ 4.5). The in vitro cytotoxicity study against two human pancreatic cancer cell lines (PANC-1 and Mia PaCa-2) demonstrated increased growth inhibition with the nanoparticle formulation compared to pure CL alone [17].

The main objectives of this study were 1 ) to optimize the preparation of CL-SFNP; 2 ) to develop and validate a HPLC method for the analysis of CL in rat plasma; and 3) to determine the pharmacokinetic profile of optimized formulation of CL-SFNP in comparison to pure CL in male Sprague-Dawley rats.

\section{Results}

\subsection{Preparation of Silk Fibroin Solution}

Concentration of the SF solution was measured by freeze-drying $5 \mathrm{~mL}$ of the solution (in triplicate) followed by weighing the material. The concentration of the solution was $4.64 \pm 0.02 \mathrm{mg} / \mathrm{mL}$, which was 
further diluted with deionized water to $2 \mathrm{mg} / \mathrm{mL}$ to give the optimized concentration for nanoparticle formation as previously reported [17]. Consistency in SF solution preparation was optimized by a stirring time of $1 \mathrm{~h}$ during the hydrolysis step of the SF material.

\subsection{Optimized Preparation of CL-Loaded Silk Fibroin Nanoparticles}

\subsubsection{Varying Storage Times at $-20{ }^{\circ} \mathrm{C}$}

Storage time affects the formation and stability of SFNP [18,19]. For this study, a wider range of storage times were evaluated. The percent yield of the lyophilized CL-SFNP product was the main indicator of quantifying the effect of different storage times at $-20^{\circ} \mathrm{C}$. The size and zeta-potential were measured to characterize the nanoparticles. Table 1 shows the percent yield, size, and zeta-potential that corresponds to eight different storage times $(1,2,4,8,16,20,24$, and $48 \mathrm{~h})$. Low percent yields were observed for storage times of 1,2 , and $48 \mathrm{~h}(1.52 \pm 0.5 \%, 3.89 \pm 1.3 \%$, and $4.54 \pm 2.1 \%$, respectively), with significantly larger size for 1 and $48 \mathrm{~h}(1390.2 \pm 84 \mathrm{~nm}$ and $4809.9 \pm 7132.2 \mathrm{~nm}$, respectively). Higher percent yields were observed for storage times from 4 to $20 \mathrm{~h}$ with the highest yield of $35.5 \% \pm 7.5 \%$ at $20 \mathrm{~h}$.

Table 1. Effect of varying storage times and rotation speeds on percentage yield and size $(n=3)$.

\begin{tabular}{cccc}
\hline Hours in $\mathbf{- 2 0} \mathbf{~}^{\mathbf{C}}$ & Rotation Speed $(\mathbf{r p m})$ & Yield $\mathbf{( \% )}(\mathbf{n}=\mathbf{3})$ & Size $(\mathbf{n m}) \mathbf{( n = 3 )}$ \\
\hline 1 & 750 & $1.52 \pm 0.5$ & $1390.2 \pm 847.6$ \\
2 & 750 & $3.89 \pm 1.3$ & $424.1 \pm 32.4$ \\
4 & 750 & $33.3 \pm 6.9$ & $266.1 \pm 2.3$ \\
8 & 750 & $26.9 \pm 8.1$ & $321.2 \pm 5.5$ \\
16 & 750 & $31.9 \pm 3.1$ & $277.1 \pm 1.5$ \\
20 & 750 & $35.5 \pm 7.5$ & $333.5 \pm 16.5$ \\
24 & 750 & $25.2 \pm 2.9$ & $312.1 \pm 1.4$ \\
48 & 750 & $4.54 \pm 2.1$ & $4809.9 \pm 7132.2$ \\
20 & 625 & $27.1 \pm 4.7$ & $256.2 \pm 13.1$ \\
20 & 750 & $35.5 \pm 7.5$ & $333.5 \pm 38.8$ \\
20 & 875 & $18.4 \pm 7.2$ & $292.7 \pm 28.1$ \\
\hline
\end{tabular}

\subsubsection{Varying Rotation Speeds}

Stirring speed has an impact on $\beta$-sheet formation, which is responsible for the stability and mechanical properties of the SF material and in turn may affect the formation and stability of nanoparticles [13,16]. The stirring speed during the addition of $2 \mathrm{mg} / \mathrm{mL} \mathrm{CL}$ into $2 \mathrm{mg} / \mathrm{mL} \mathrm{SF}$ was observed at a slightly lower speed of $625 \mathrm{rpm}$ and a higher speed of $875 \mathrm{rpm}$ with $20 \mathrm{~h}$ as the selected storage time at $-20^{\circ} \mathrm{C}$. The percent yield for stirring speed $625 \mathrm{rpm}$ was $27.1 \% \pm 4.7 \%$, which is slightly lower than that of the set speed of $750 \mathrm{rpm}$. Increasing the speed to $875 \mathrm{rpm}$ resulted in a much lower percent yield of $18.4 \% \pm 7.2 \%$ compared to that for the $750 \mathrm{rpm}$ stirring speed (Table 1 ).

\subsubsection{Inter-Day Evaluation of Optimized Formulation}

Storage time of $20 \mathrm{~h}$ at $-20^{\circ} \mathrm{C}$ and a stirring speed of $750 \mathrm{rpm}$ were selected based on the product yield. Inter-day evaluation was performed on three consecutive days with full characterization of the nanoparticles as given (Table 2). The product yield was $26.5 \% \pm 8.7 \%, 31.1 \% \pm 16.9 \%$, and $34.3 \% \pm 14.5 \%$ for days 1, 2, and 3, respectively. Therefore, CL-SFNP could be prepared consistently using the optimized procedures. The general flow chart of CL-SFNP preparation is shown in Figure 2. 
Table 2. Inter-day evaluation for physical properties of optimized formulation $(n=3)$.

\begin{tabular}{cccccc}
\hline Day & Yield $\mathbf{( \% )}$ & Size $(\mathbf{n m})$ & Zeta-Potential $(\mathbf{m V})$ & Encapsulation Efficiency $(\%)$ & Drug Loading $(\boldsymbol{\mu g} / \mathbf{m g})$ \\
\hline 1 & $26.5 \pm 8.7$ & $291.1 \pm 22.1$ & $-25.2 \pm 3.2$ & $81.4 \pm 8.2$ & $97.2 \pm 28.4$ \\
2 & $31.7 \pm 16.9$ & $298.9 \pm 20.7$ & $-19.5 \pm 5.4$ & $83.2 \pm 14.7$ & $80.9 \pm 20.0$ \\
3 & $34.3 \pm 14.5$ & $299.5 \pm 20.3$ & $-22.3 \pm 0.9$ & $79.3 \pm 11.3$ & $80.9 \pm 4.2$ \\
\hline
\end{tabular}

CL was dissolved in an acetone: ethanol ( $3: 2 \mathrm{v} / \mathrm{v})$ mixture

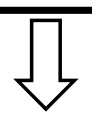

Introduced in SF solution dropwise under gentle stirring

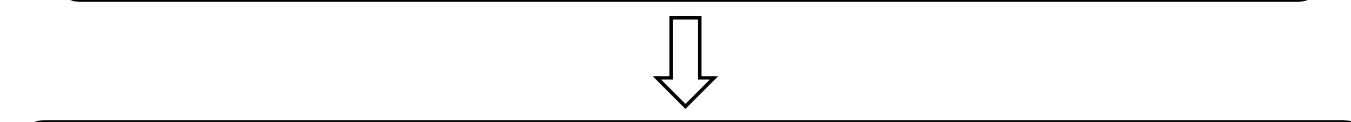

Resulting suspension was incubated in a refrigerator at $-20^{\circ} \mathrm{C}$ for next $1-48 \mathrm{~h}$ and defrosted quickly in a water bath $\left(30-35^{\circ} \mathrm{C}\right)$

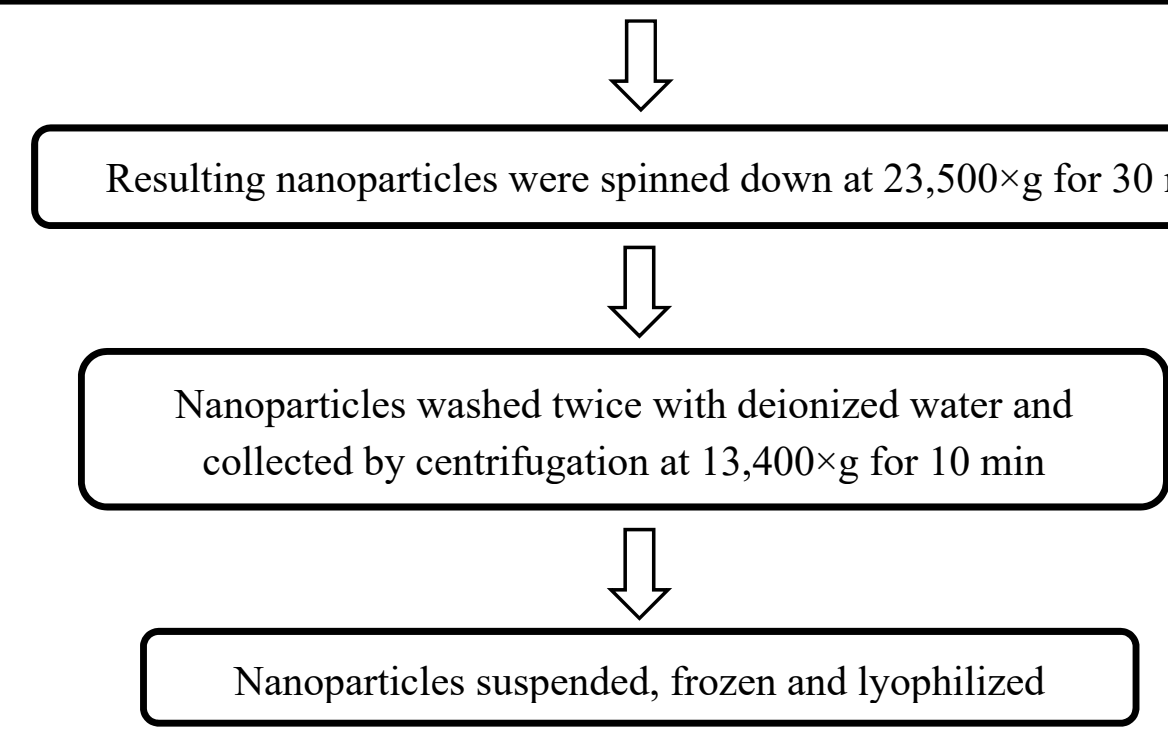

Figure 2. Flow diagram of preparation of CL encapsulated silk fibroin (SF) nanoparticles.

\subsection{Analytical Method Validation}

\subsubsection{Chromatographic Conditions}

Pristimerin (Figure 1) was selected as the internal standard (IS) based on its similarity in structure and ultraviolet (UV) absorption spectrum to CL. The maximum UV absorbance of CL and pristimerin was at $425 \mathrm{~nm}$ [20]. The mobile phase was a mixture of acetonitrile and $1 \%$ phosphoric acid $\left(\mathrm{H}_{3} \mathrm{PO}_{4}\right)$ $(85: 15 \mathrm{v} / \mathrm{v})$. Acidification of the mobile phase was to prevent $\mathrm{CL}$ from ionizing and improve the peak shape. Retention times of CL and pristimerin were 4.9 and 9.4 min, respectively.

\subsubsection{Selectivity and Specificity}

The potential inferences of biological matrix with both the analyte and IS were assessed in blank rat plasma, rat plasma spiked with IS, CL, or SFNP. All samples were processed for HPLC analysis with no interference observed (Figure 3). 


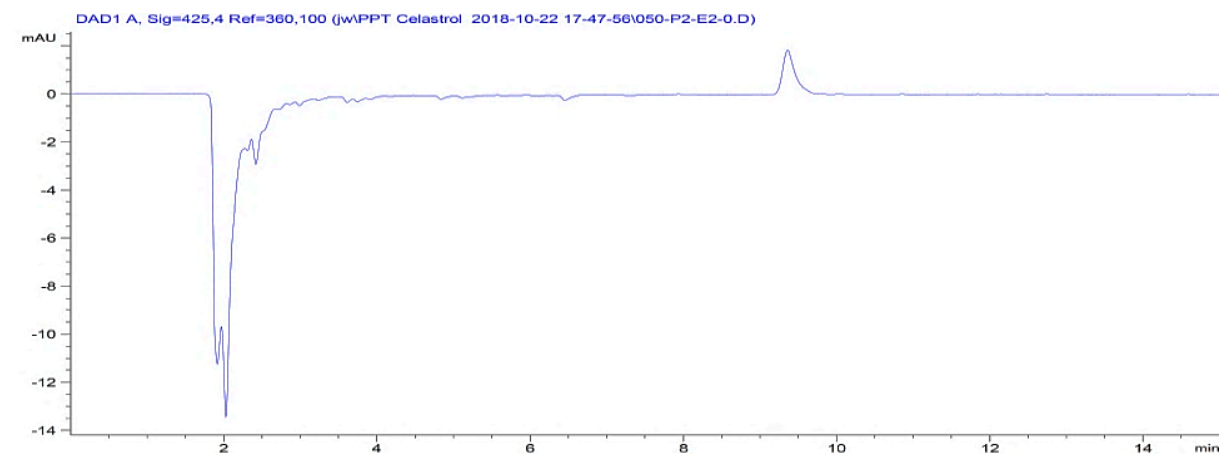

(a)

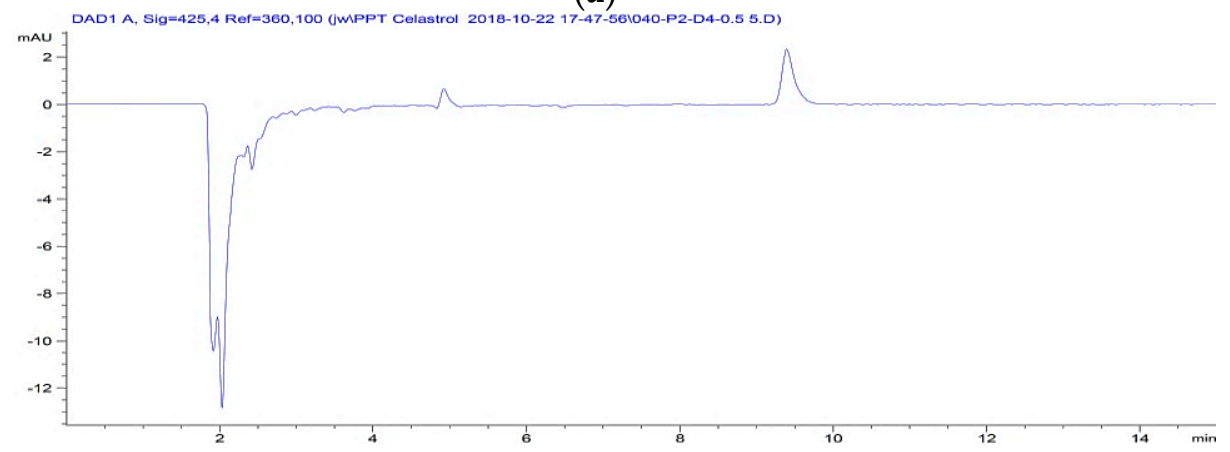

(b)

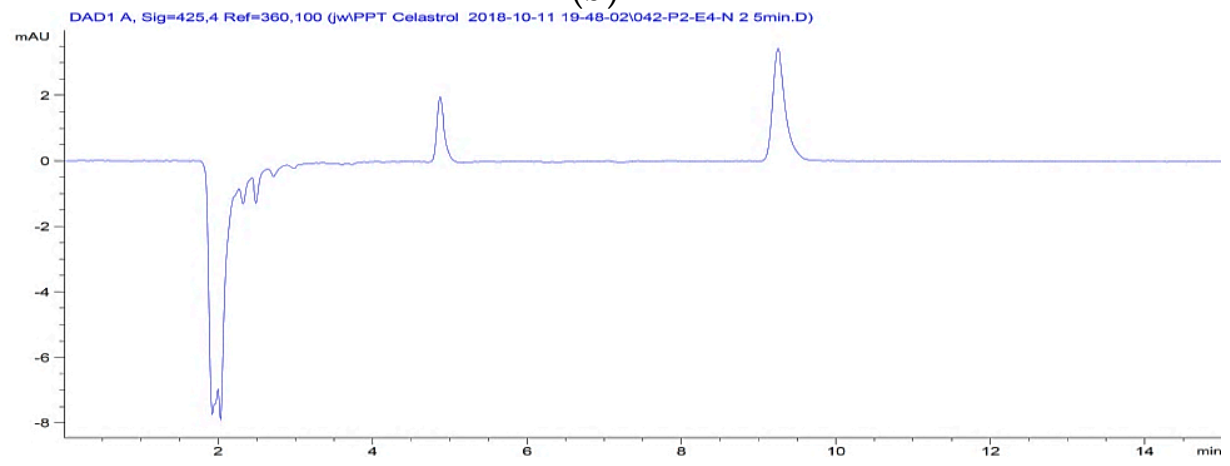

(c)

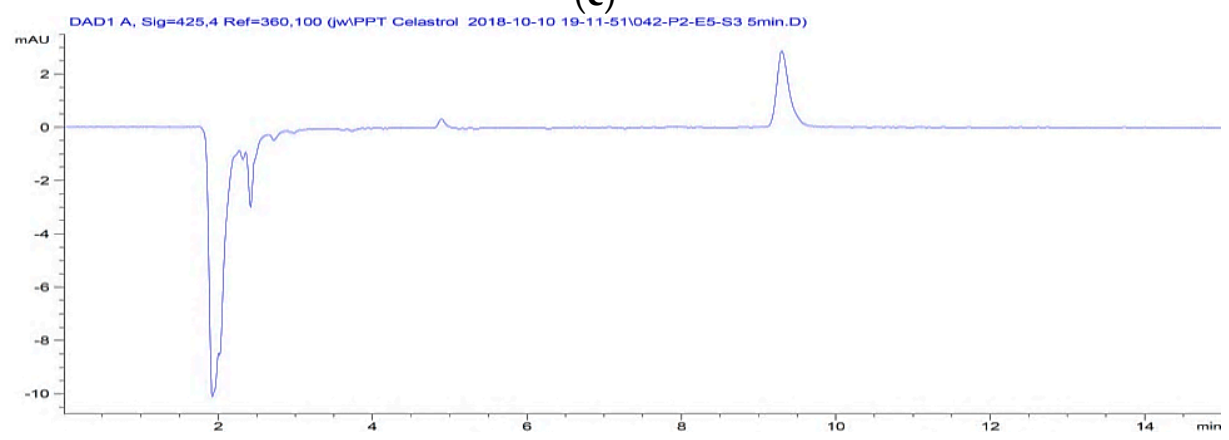

(d)

Figure 3. Chromatograms of processed samples of (a) Blank plasma spiked IS (2 $\mu \mathrm{g} / \mathrm{mL})$ (b) Rat plasma spiked with CL $(0.5 \mu \mathrm{g} / \mathrm{mL})$ and IS $(2 \mu \mathrm{g} / \mathrm{mL})$ (c) Rat plasma at $5 \mathrm{~min}$ after IV dosing of CL-SFNP $(1 \mathrm{mg} / \mathrm{kg})$. (d) Rat plasma at $5 \mathrm{~min}$ after IV dosing of CL-solution $(1 \mathrm{mg} / \mathrm{kg})$.

\subsubsection{Linearity and Sensitivity}

The calibration curve demonstrated linearity in the concentration range of $0.05-5 \mu \mathrm{g} / \mathrm{mL}$, with an $R^{2}$ value greater than 0.99 . Sensitivity was defined as the lowest concentration that could be quantified 
in accordance to precision and accuracy [21]. The lowest level of quantification (LLOQ) was found at $0.05 \mu \mathrm{g} / \mathrm{mL}$.

\subsubsection{Precision and Accuracy}

The intra- and inter-day precision and accuracy were evaluated at the lower limit of quantification (LLOQ), low quality control (LQC), medium quality control (MQC), and high quality control (HQC) concentrations as indicated in Table 3. The intra-/inter-day precision of the quality control (QC) samples defined by relative standard deviation (RSD) ranged from $2.60 \%$ to $14.33 \%$ and $1.94 \%$ to $17.60 \%$ for LLOQ samples, which was within the limit of the acceptance criteria of $\%$ RSD $\leq 15 \%$ for QC samples and $\leq 20 \%$ for LLOQ. The accuracy ranged from $90.22 \%$ to $114.26 \%$ for QC samples and $110.75 \%$ to $119.28 \%$ for LLOQ, where the values did not deviate from the nominal concentration by $\pm 15 \%$ for QC samples and $\pm 20 \%$ for LLOQ [21].

Table 3. Intra-day, inter-day precision, and accuracy of nominal concentrations.

\begin{tabular}{ccccc}
\hline $\begin{array}{c}\text { Nominal } \\
\begin{array}{c}\text { Concentration } \\
(\mu \mathrm{g} / \mathrm{mL})\end{array}\end{array}$ & \multicolumn{2}{c}{ Intra-Day $(\mathbf{n}=\mathbf{5})$} & \multicolumn{2}{c}{ Inter-Day $(\mathbf{n}=\mathbf{3})$} \\
\cline { 2 - 5 } & $\begin{array}{c}\text { Precision } \\
(\% \mathbf{R S D})\end{array}$ & $\begin{array}{c}\text { Accuracy } \\
\mathbf{( \% )}\end{array}$ & $\begin{array}{c}\text { Precision } \\
(\mathbf{\%} \text { RSD) }\end{array}$ & $\begin{array}{c}\text { Accuracy } \\
\mathbf{( \% )}\end{array}$ \\
\hline $0.05(\mathrm{LLOQ})$ & 17.6 & 110.7 & 10.8 & 116.3 \\
0.1 & 4.6 & 114.2 & 7.5 & 107.8 \\
0.5 & 4.3 & 92.2 & 10.8 & 97.2 \\
5.0 & 5.3 & 101.5 & 4.7 & 95.8 \\
\hline
\end{tabular}

RSD—relative standard deviation; LLOQ—lowest level of quantification.

\subsubsection{Recovery}

The method used for extraction recovery was protein precipitation with acetonitrile. Table 4 shows the percent recovery of CL and IS. The recovery of CL was above $40 \%$ ranging from $40.95 \%$ to $67.43 \%$, and that of the IS was $75.28 \%$. Although the recovery of CL was relatively low, it was reproducible, which meets FDA guideline [21].

Table 4. Recovery of CL and internal standard (IS) from rat plasma $(n=5)$.

\begin{tabular}{ccc}
\hline Analyte & Concentration $(\mu \mathrm{g} / \mathrm{mL})$ & Recovery (\%) \\
\hline CL & 0.1 & $67.4 \pm 5.3$ \\
& 0.5 & $42.0 \pm 2.7$ \\
Pristimerin (IS) & 5.0 & $41.0 \pm 5.5$ \\
\hline
\end{tabular}

\subsection{Pharmacokinetic Study}

From previous studies, CL has demonstrated poor pharmacokinetic profile with low bioavailability and rapid clearance [3]. Nanocarriers have been used to deliver problematic pharmaceuticals, by improving drug absorption, distribution, metabolism, and excretion [22,23]. In this study, the optimized CL-SFNP was evaluated compared to pure CL in solution following IV administration. The plasma concentration-time curves shown in Figure 4 demonstrated an improvement in pharmacokinetic behavior upon SF nanocapsulation. The CL initial concentration $\left(C_{0}\right)$ following CL-SFNP administration was four times higher than that of $C L$ in solution in rats. Similarly, the total area under the curve $\left(\mathrm{AUC}_{0 \text {-inf }}\right)$ value with the nanoformulation was 2.5 times higher and had a mean residence time (MRT) that was twice as long compared to those with CL in solution. The relevant pharmacokinetic parameters are shown in Table 5. The circulation time of CL in blood was longer with the NP formulation when compared to that with CL in solution. Our findings align with previous reports on the improvement of pharmacokinetic profiles with various CL-NPs [20,24-26]. 


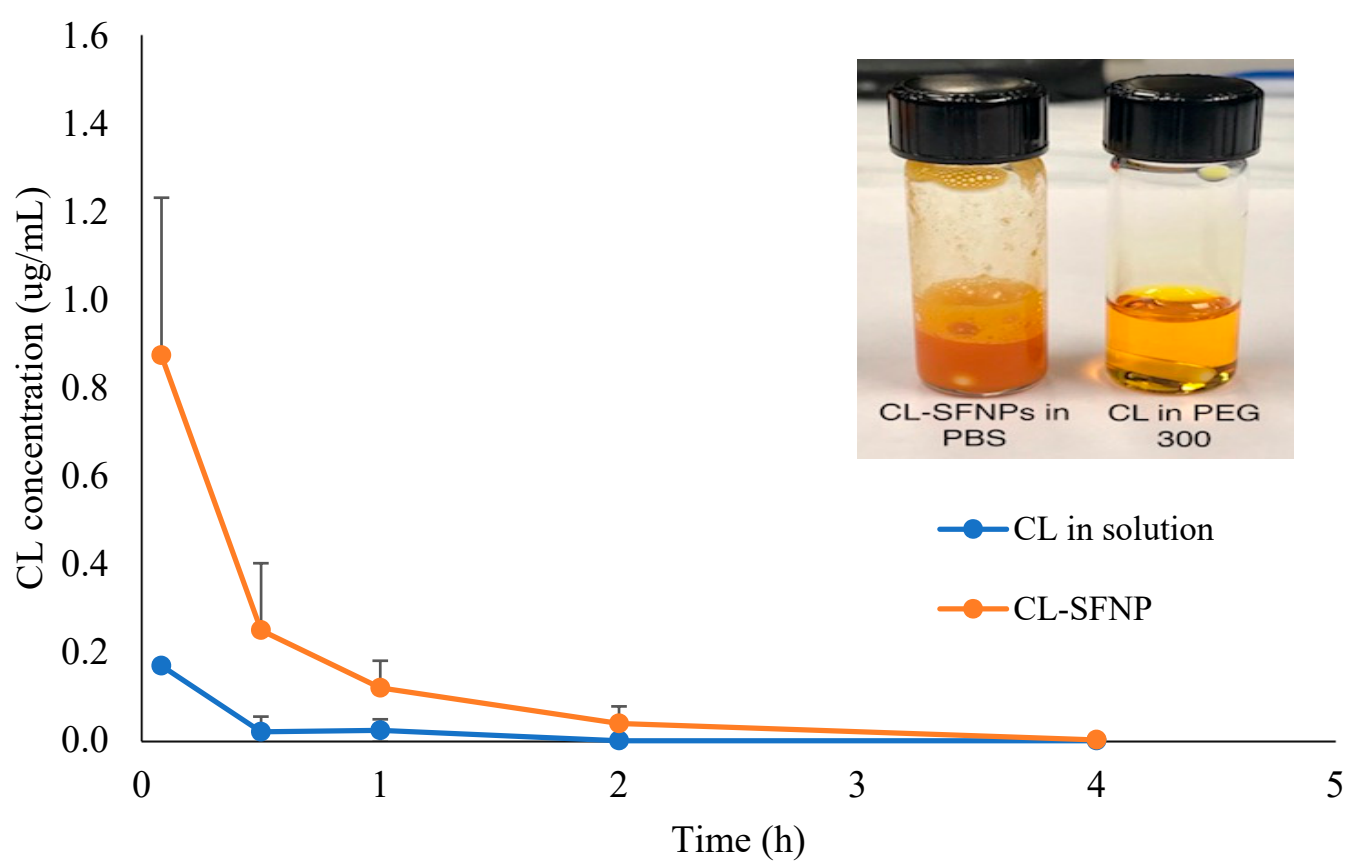

Figure 4. CL plasma concentration-time profiles in rats following intravenous (IV) administration of $\mathrm{CL}$ and celastrol-loaded silk fibroin nanoparticles (CL-SFNP). The insert shows the physical appearance of the respective formulations.

Table 5. Pharmacokinetic parameters of CL following single intravenous dose of pure CL and CL-SFNP at $1 \mathrm{mg} / \mathrm{kg}(\mathrm{n}=3)$.

\begin{tabular}{ccc}
\hline Parameters & CL in PEG 300 & CL-SFNP \\
\hline $\mathrm{C}_{0}\left(\mu \mathrm{g} \mathrm{mL}^{-1}\right)$ & 0.25 & 1.09 \\
$\mathrm{AUC}_{0-\text { inf }}\left(\mu \mathrm{g} \mathrm{h} \mathrm{mL}^{-1}\right)$ & $0.18 \pm 0.31$ & $0.47 \pm 0.20$ \\
$\mathrm{CL}\left(\mathrm{mL} \mathrm{h}^{-1}\right)$ & $1.78 \pm 0.94$ & $0.71 \pm 0.33$ \\
$\mathrm{MRT}(\mathrm{h})$ & $0.26 \pm 0.44$ & $0.51 \pm 0.07$ \\
\hline
\end{tabular}

\section{Discussion}

The pharmacokinetic properties of CL have rarely been reported mainly because of its low concentration in plasma which is due to low solubility (Song et al., 2011) and extensive in vivo metabolism [3]. CL is undissolvable in water, with a $\log P$ of 5.63 which qualifies CL as a Biopharmaceutics Classification System (BCS) Class IV-type drug, suggesting CL may be poorly absorbed by the body [27]. To overcome these limitations, nanotechnology has been employed to improve both physiochemical and pharmacokinetic properties of potential compounds. The protein material employed for this study was SF, which is known for its exceptional mechanical durability and biocompatibility. The fabrication of CL-SFNP was generated by the desolvation method, where the drug dissolved in organic solvent was introduced, dropwise, into SF solution under stirring.

Before the application of CL-loaded SF nanoparticles for in vivo evaluation, adjustments were made to optimize the product in regard to varying storage time and rotation speed, followed by inter-day evaluation of the finalized formulation. A stable SF solution that yielded greater NP product was observed when the SF material was stirred for $1 \mathrm{~h}$ as opposed to $30 \mathrm{~min}$. Of the range of storage times, $20 \mathrm{~h}$ was selected as it presented the most percent yield of CL-SFNP product of $35.5 \% \pm 7.5 \%$, despite the slightly larger size than at $4 \mathrm{~h}$. CL-SFNP was negatively charged. A stirring speed of $750 \mathrm{rpm}$ was found favorable and was chosen to be suitable for further studies. The characteristics of the nanoparticles were consistent from three batches prepared on three consecutive days. The optimized procedure for CL-SFNP preparation was used to generate product that was used in the pharmacokinetic study. 
A high-performance liquid chromatography method was developed and validated for the analysis of CL in rat plasma in terms of selectivity, linearity, intra-/inter-day precision and accuracy, and recovery. The HPLC method was validated using Food and Drug Administration (FDA) guidelines with regard to linearity where the $\mathrm{R}^{2}$ value was greater than 0.99 , intra-/inter-day precision and accuracy values with a percent deviation below $15 \%$ for QC samples and $20 \%$ for LLOQ, and a consistent recovery. The validated method was applied for the analysis of CL in plasma to evaluate the pharmacokinetic behavior of CL-SFNP in comparison with CL solution.

The pharmacokinetic profile was improved with CL-loaded SF nanoparticles compared to pure $\mathrm{CL}-$ which was observed following IV administration. The initial concentration of CL nanoparticles was four times higher than that of CL in solution and CL-SFNP demonstrated longer mean residence time. Future research will focus on the evaluation of bioactivities of CL-SFNP in comparison with $\mathrm{CL}$, with a long-term aim of developing CL-SFNP for treating diseases such as PDAC, autoimmune diseases, and obesity.

\section{Materials and Methods}

\subsection{Chemicals and Reagents}

Celastrol (CL) was purchased from Chengdu Biopurify Phytochemicals Ltd. (Chengdu, China). Cocoons from B. mori silkworms were kindly supplied by Tongxiang Mulberry Silk Base of Zhejiang Province (Tongxiang, China). A dialysis membrane with a cut-off of 7000 Da molecular weight cut-off (MWCO) was obtained from Viskase Companies, Inc. (Chicago, USA). Pristimerin purchased from PI \& PI TECH (Macon, GA, USA). HPLC-grade acetonitrile and methanol used for the analysis were purchased from VWR (Randor, PA, USA). HPLC-grade phosphoric acid was obtained from Fisher Scientific (Pittsburgh, PA, USA). Ultrapure water was obtained from Millipore equipment (Millipore, Bedford, MA, USA). Rat plasma was purchased from Innovative Research (Novi, MI, USA). Polyethylene glycol 300 was purchased from Spectrum (Gardena, CA, USA). Dulbecco's phosphate buffered saline was purchased from Genesse Scientific (San Diego, CA, USA).

\subsection{Preparation of Silk Fibroin Solution}

One gram of cut B. mori cocoon was degummed twice in $0.02 \mathrm{M}$ sodium carbonate $\left(\mathrm{Na}_{2} \mathrm{CO}_{3}\right)$ for $30 \mathrm{~min}$ with rinses with deionized water between degumming steps. The extracted SF was allowed to dry overnight at room temperature. Dried SF was then hydrolyzed in $50 \mathrm{~mL}$ of a ternary system containing calcium chloride dihydrate $\left(\mathrm{CaCl}_{2} \cdot 2 \mathrm{H}_{2} \mathrm{O}\right)$ :ethanol:water (molar ratio of 1:2:6) under constant stirring for $1 \mathrm{~h}$ until SF was completely dissolved, followed by an additional $3 \mathrm{~h}$ without stirring with temperature maintained at $75{ }^{\circ} \mathrm{C}$. The hydrolyzed SF was transferred into $50 \mathrm{~mL}$ centrifuge tubes and centrifuged for $15 \mathrm{~min}$ at $4500 \mathrm{rpm}$ to remove any undissolved material and the supernatant was subsequently dialyzed against deionized water under gentle stirring $(250 \mathrm{rpm})$ for the next $72 \mathrm{~h}$. Water was changed after 1 and $3 \mathrm{~h}$ on day 1 and twice a day for day 2 and day 3 . The dialyzed SF solution was collected and centrifuged for $15 \mathrm{~min}$ at $14,000 \mathrm{rpm}$ to remove any nanoparticles that might have formed during the dialysis. The supernatant was collected and a $5 \mathrm{~mL}$ sample was placed into pre-weighed vials, and lyophilized to determine the concentration of the SF solution $[17,18]$. Based on the result, the $\mathrm{SF}$ solution was diluted to $2 \mathrm{mg} / \mathrm{mL}$.

\subsection{Preparation of CL-Loaded Silk Fibroin Nanoparticles}

For the preparation of CL-SFNP, $0.4 \mathrm{~mL}$ of $2 \mathrm{mg} / \mathrm{mL}$ of CL (CL) dissolved in acetone:ethanol $(3: 2 \mathrm{v} / \mathrm{v})$ was added dropwise into $4 \mathrm{~mL}$ of $2 \mathrm{mg} / \mathrm{mL} \mathrm{SF}$ solution under gentle stirring at different stirring speeds of 625,750 , and $875 \mathrm{rpm}$. The addition of CL into SF solution was completed in the span of $2 \mathrm{~min}$ and the mixture was allowed to stir for $10 \mathrm{~min}$, followed by sonication for $3 \mathrm{~min}$. The suspension was then stored at $-20{ }^{\circ} \mathrm{C}$ for a fixed time and quickly thawed at $35^{\circ} \mathrm{C}$. The thawed suspension was then subjected to ultracentrifugation at 14,200 rpm for $30 \mathrm{~min}$. The pellet was separated and washed 
twice with deionized water and the nanosuspension was further dispersed by ultrasound processor set at $10 \%$ amplitude for $1 \mathrm{~min}$. The suspension was then frozen at $-80{ }^{\circ} \mathrm{C}$ followed by lyophilization. The lyophilized product was weighed and percent recovery was determined $[17,28,29]$. All the steps are shown in Figure 2.

\subsection{Nanoparticle Characterization}

\subsubsection{Size and Zeta-potential}

To one milligram of freeze-dried CL-SFNP was added $2 \mathrm{~mL}$ of deionized water to obtain a concentration of $0.5 \mathrm{mg} / \mathrm{mL}$ of CL-SFNP. The suspension was then dispersed by an ultrasound processor set at $10 \%$ amplitude for $5 \mathrm{~min}$. The dispersed suspension was further diluted (1:5) with deionized water, followed by analysis with NanoBrook $^{\circledR}$ (Brookhaven Instruments Corporation, NY, USA) to measure the size of CL-SFNP. The surface charge of CL-SFNP was measured by Zetasizer ${ }^{\circledR}$ (Nano ZS90, Malvern, Malvern Instruments Ltd., Worcestershire, UK).

\subsubsection{Encapsulation Efficiency and Drug Loading}

To measure the drug content of CL in SF nanoparticles, $2 \mathrm{~mL}$ of acetone:ethanol $(3: 2 \mathrm{v} / \mathrm{v})$ was added to $0.5 \mathrm{mg}$ of freeze-dried product to dissolve the drug. The mixture was centrifuged at $14,000 \mathrm{rpm}$ for $15 \mathrm{~min}$ and the supernatant was collected and used for HPLC analysis. As for the encapsulation efficiency, the supernatant and washes that were collected during the CL-SFNP preparation, was centrifuged at 14,000 rpm for $15 \mathrm{~min}$ and the supernatant was used for HPLC analysis.

Encapsulation efficiency and drug loading was measured by Agilent 1260 infinity HPLC systems (Agilent Technologies, Palo Alto, CA, USA). Chromatographic separation was carried out on an Accucore $\mathrm{XL} \mathrm{C}_{18}$ column $(250 \mathrm{~mm} \times 4.6 \mathrm{~mm}, 4 \mu \mathrm{m})$. Analysis was performed with UV detection, using a diode array detector (DAD) set at $425 \mathrm{~nm}$ for CL. The mobile phase consisted of $1 \%(\mathrm{v} / \mathrm{v})$ phosphoric acid (A) and methanol (B), programmed for a gradient elution, where the mobile phase (B) increased from $58 \%$ to $95 \%$ during $4 \mathrm{~min}$ and remained at $95 \%$ for $8 \mathrm{~min}$. The gradient elution ran at a flow rate of $1 \mathrm{~mL} / \mathrm{min}$ and the acquisition time was $12 \mathrm{~min}$. Encapsulation efficiency and drug loading were calculated using the following equations.

$$
\begin{aligned}
& \text { Encapsulation ef ficiency } \\
& =\frac{\text { Total weight of feeding drug }(\mathrm{mg})-\text { Amount of drug analyzed by HPLC method }(\mathrm{mg})}{\text { Total weight of feeding drug }(\mathrm{mg})} \times 100 \% \\
& \text { Drug loading equation }=\frac{\text { Amount of drug analyzed by HPLC method }(\mu g)}{\text { Weight of nanoparticles }(m g)}
\end{aligned}
$$

\subsection{Optimized Formulation of CL-Loaded Silk Fibroin Nanoparticles}

\subsubsection{Varying Storage Times at $-20^{\circ} \mathrm{C}$}

To investigate a suitable storage time for CL-SFNP at $-20{ }^{\circ} \mathrm{C}$, the preparations were stored at $-20^{\circ} \mathrm{C}$ for $1,2,4,8,16,20,24$, and $48 \mathrm{~h}$. After storage for the indicated times the CL-SFNP were further purified following the procedures mentioned above.

\subsubsection{Varying Rotation Speeds}

The rotation speed at time of loading CL into SF solution was set at 625,750 , or $875 \mathrm{rpm}$. The drug-loaded nanoparticles were stored for the optimized time of $20 \mathrm{~h}$ at $-20{ }^{\circ} \mathrm{C}$. 


\subsubsection{Inter-Day/Intra-Day Evaluation of the Finalized Formulation}

To confirm the integrity of the finalized formulation of CL-SFNP, the product yield was observed for three consecutive days and was done in triplicate. The lyophilized product was weighed and further characterized in terms of size, zeta-potential, encapsulation efficiency, and drug loading.

\subsection{Development and Validation of Method for Preclinical Studies}

To ensure that the HPLC method developed for the analysis of CL in rat plasma for pharmacokinetic studies was reliable and reproducible, the method was validated in terms of specificity and selectivity, linearity, precision and accuracy, recovery, and stability [21].

\subsubsection{Chromatographic Conditions}

Chromatographic separation was carried out on an Accucore XL C ${ }_{18}$ column $(250 \mathrm{~mm} \times 4.6 \mathrm{~mm}$, $4 \mu \mathrm{m}$ ) using an Agilent 1260 Infinity HPLC system. Analysis was performed with ultraviolet (UV) detection using a diode array detector (DAD) set at $425 \mathrm{~nm}$ for CL and pristimerin (IS). The mobile phase consisted of acetonitrile and $1 \% \mathrm{H}_{3} \mathrm{PO}_{4}(85: 15 \mathrm{v} / \mathrm{v})$, with a flow rate set at $1 \mathrm{~mL} / \mathrm{min}$ and column temperature set at $25^{\circ} \mathrm{C}$. The acquisition time was $15 \mathrm{~min}$.

\subsubsection{Preparation of Standard and Quality Controls}

$\mathrm{CL}$ and internal standard stock solutions were prepared at concentration of $1 \mathrm{mg} / \mathrm{mL}$ in methanol. The CL calibration standard concentrations of $0.05,0.1,0.2,0.5,1$, and $5 \mu \mathrm{g} / \mathrm{mL}$ were prepared in blank rat plasma. Quality control (QC) samples were set at low $(0.1 \mu \mathrm{g} / \mathrm{mL})$, medium $(0.5 \mu \mathrm{g} / \mathrm{mL})$, and high $(5 \mu \mathrm{g} / \mathrm{mL})$ concentrations, and were prepared the same as standard concentrations. All standard and quality control samples were spiked with $10 \mu \mathrm{L}$ IS of concentration $20 \mu \mathrm{g} / \mathrm{mL}$.

\subsubsection{Preparation of Plasma Samples}

Plasma samples (100 $\mu \mathrm{L}$ each) were spiked with $10 \mu \mathrm{L}$ of IS. The spiked plasma samples were extracted using the protein precipitation method with $400 \mu \mathrm{L}$ of acetonitrile. The precipitated sample was vortexed for $1 \mathrm{~min}$ and then underwent centrifugation for $5 \mathrm{~min}$ at 14,000 rpm. The supernatant was transferred into glass tubes and the solvent was removed by evaporation with a gentle stream of air for $2.5 \mathrm{~h}$. The dried residue was reconstituted with $50 \mu \mathrm{L}$ of the mobile phase by vortexing for $1 \mathrm{~min}$ and centrifuging for $5 \mathrm{~min}$ at 14,000 rpm. Supernatant volume of $35 \mu \mathrm{L}$ was collected and transferred into vials for HPLC analysis.

\subsubsection{Specificity and Selectivity}

Absence of interference with the matrix and the analyte was ascertained for six sources. Selectivity was evaluated by injecting the blank plasma, plasma spiked with CL or IS, plasma spiked with both CL and IS, or plasma spiked with blank SFNP formulation.

\subsubsection{Linearity and Sensitivity}

Linearity was evaluated by injecting a standard curve with six concentrations of CL ranging from 0.05 to $5 \mu \mathrm{g} / \mathrm{mL}$. A calibration curve was constructed using CL peak area to IS peak area (ratio CL/IS) and plotting it versus the nominal concentrations of CL. Linearity of the curve was generated by using linear least square regression $\left(\mathrm{R}^{2}\right)$ analysis. Sensitivity was defined by the lowest concentration of the curve that can be measured and quantified in terms of precision and accuracy. The CL concentration of $0.05 \mu \mathrm{g} / \mathrm{mL}$ was determined as the LLOQ.

\subsubsection{Precision and Accuracy}

Standard and quality control samples were evaluated in terms of intra-day precision and accuracy in replicates of five on a single day and for inter-day precision and accuracy over three consecutive 
days. Relative standard deviation (RSD\%) was determined for precision and was acceptable of all quality control (QC) samples which did not exceed $15 \%$ and $20 \%$ for LLOQ samples under the acceptance criteria set by FDA guidelines. Accuracy was determined by comparing the calculated concentrations derived from the equation form the calibration curve to the nominal concertation. The acceptance criteria for accuracy was the mean values of calculated concentration compared to nominal concentration fell within $\pm 15 \%$ for QC samples and $\pm 20 \%$ for LLOQ.

\subsubsection{Recovery}

The extraction recovery of CL and IS in rat plasma was compared to non-extracted standard solution containing same amount of both analyte and internal standard. The recovery was evaluated at all levels of QC samples in replicates of five $(0.1,0.5$, and $5 \mu \mathrm{g} / \mathrm{mL})$ for CL and for IS concentration of $2 \mu \mathrm{g} / \mathrm{mL}$. The process to determine extraction has been mentioned above and compared with non-extracted samples represented as analyte and IS in solution.

\subsection{Pharmacokinetics}

The study on animal protocol was approved by Western University of Health Sciences, Pomona Institutional Animal Care and Use Committee (IACUC). Male Sprague-Dawley rats with pre-cannulated jugular vein were administered an intravenous (IV) dose of $1 \mathrm{mg} / \mathrm{kg}$. Rats were separated into two groups $(\mathrm{n}=3)$ and were administered pure CL solution in PEG 300 or CL-SFNP suspension in phosphate buffer solution (PBS) (Figure 4) into the pre-cannulated jugular vein. After IV administration, $200 \mu \mathrm{L}$ of blood was collected into pre-heparinized tubes at $0.083,0.5,1,2,4,6,8,12$, and $24 \mathrm{~h}$. After $24 \mathrm{~h}$ the rats were euthanized with $30 \%$ isoflurane. Collected blood was then centrifuged for $15 \mathrm{~min}$ at $14,000 \mathrm{rpm}$. After centrifugation $110 \mu \mathrm{L}$ of plasma was collected and transferred into Eppendorf tubes, and then stored at $-20^{\circ} \mathrm{C}$ until analysis. The pharmacokinetic parameters were generated by non-compartmental analysis using PK Functions for Microsoft Excel [30].

\section{Conclusions}

The optimization of CL-SFNP with respects to storage time at $-20^{\circ} \mathrm{C}$ and rotation speeds was able to be evaluated where a greater product yield of CL-SFNP was collected and the selected parameters were subjected to pharmacokinetic analysis. The HPLC method was developed and validated and further used in the analysis of plasma concentration of CL for pharmacokinetic studies. The CL-SFNP demonstrated an improvement of the pharmacokinetic profile of CL compared to pure CL.

Author Contributions: Conceptualization, J.W.; methodology, B.D., M.A.W., F.O. and A.P.; validation, F.O. and A.P.; investigation, F.O., A.P. and J.L.; data curation, F.O., A.P. and S.K. writing-original draft preparation, F.O., A.P. and S.K.; writing-review and editing, S.K., S.P., G.B. and J.W.

Funding: This research received no external funding.

Conflicts of Interest: The authors declare no conflict of interest.

\section{References}

1. Adamska, A.; Domenichini, A.; Falasca, M. Pancreatic Ductal Adenocarcinoma: Current and Evolving Therapies. Int. J. Mol. Sci. 2017, 18, 1388. [CrossRef]

2. Efferth, T.; Li, P.C.H.; Badireenath Konkimalla, V.S.; Kaina, B. From traditional Chinese medicine to rational cancer therapy. Trends Mol. Med. 2007, 13, 353-361. [CrossRef] [PubMed]

3. Zhang, J.; Li, C.Y.; Xu, M.J.; Wu, T.; Chu, J.H.; Liu, S.J.; Ju, W.Z. Oral bioavailability and gender-related pharmacokinetics of celatrol following adminstration of pure celastrol and its related tablets in rats. J. Ethnopharmacol. 2012, 144, 195-200. [CrossRef] [PubMed]

4. Liu, J.; Lee, J.; Salazar Hernandez, M.-A.; Mazitschek, R.; Ozcan, U. Treatment of obesity with celastrol. Cell 2015, 161, 999-1011. [CrossRef] 
5. Feng, X. 307-LB: IL1R1 Mediates Celastrol's Leptin-Sensitization and Antiobesity Effects. Diabetes 2019, 68 (Suppl. S1), 307-LB. [CrossRef]

6. Liu, Z.; Ma, L.; Zhou, G.-B. The main anticancer bullets of the Chinese medicinal herb, thunder god vine. Molecules 2011, 16, 5283-5297. [CrossRef] [PubMed]

7. Jiang, Q.-W.; Cheng, K.-J.; Mei, X.-L.; Qiu, J.-G.; Zhang, W.-J.; Xue, Y.-Q.; Qin, W.-M.; Yang, Y.; Zheng, D.-W.; Chen, Y.; et al. Synergistic anticancer effects of triptolide and celastrol, two main compounds from thunder god vine. Oncotarget 2015, 6, 32790-32804. [CrossRef]

8. Cascao, R.; Fonseca, J.E.; Moita, L.F. Celastrol: A spectrum of treatment opportunities in chronic diseases. Front. Med. (Lausanne) 2017, 4, 69. [CrossRef]

9. Yang, H.; Chen, D.; Cui, Q.C.; Yuan, X.; Duo, Q.P. Celastrol, a triterpene extracted from the Chinese "Thunder of God Vine" is a potent proteasome inhibitor and suppresses human prostate cancer growth in nude mice. Cancer Res. 2006, 66, 4758-4765. [CrossRef]

10. Sanna, V.; Chamcheu, J.C.; Pala, N.; Mukhtar, H.; Sechi, M.; Siddiqui, I.A. Nanoencapsulation of natural triterpenoid celastrol for prostate cancer treatment. Int. J. Nanomed. 2015, 10, 6835-6846. [CrossRef]

11. Lohcharoenkal, W.; Wang, L.; Chen, Y.C.; Rojanasakul, Y. Protein nanoparticles as drug delivery carriers for cancer therapy. Biomed. Res. Int. 2014, 2014, 180549. [CrossRef] [PubMed]

12. Numata, K.; Kaplan, D.L. Silk-based delivery systems of bioactive molecules. Adv. Drug Deliv. Rev. 2010, 62, 1497-1508. [CrossRef] [PubMed]

13. Wani, S.U.D.; Veerabhadrappa, G.H. Silk Fibroin Based Drug Delivery Applications: Promises and Challenges. Curr. Drug Targets 2018, 19, 1177-1190. [CrossRef] [PubMed]

14. Wenk, E.; Merkle, H.P.; Meinel, L. Silk fibroin as a vehicle for drug delivery applications. J. Control Release 2011, 150, 128-141. [CrossRef] [PubMed]

15. Zhao, Z.; Li, Y.; Xie, M.-B. Silk fibroin-based nanoparticles for drug delivery. Int. J. Mol. Sci. 2015, 16, 4880-4903. [CrossRef] [PubMed]

16. Cheng, Y.; Koh, L.-D.; Li, D.; Ji, B.; Han, M.-Y.; Zhang, Y.-W. On the strength of $\beta$-sheet crystallites of Bombyx mori silk fibroin. J. R. Soc. Interface 2014, 11, 20140305. [CrossRef] [PubMed]

17. Ding, B.; Wahid, M.A.; Wang, Z.; Xie, C.; Thakkar, A.; Prabhu, S.; Wang, J. Triptolide and celastrol loaded silk fibroin nanoparticles show synergistic effect against human pancreatic cancer cells. Nanoscale 2017, 9 , 11739-11753. [CrossRef]

18. Chen, M.; Shao, Z.; Chen, X. Paclitaxel-loaded silk fibroin nanospheres. J. Biomed. Mater. Res. A 2012, 100, 203-210. [CrossRef]

19. Nam, J.; Park, Y.H. Morphology of Regenerated Silk Fibroin: Effects of Freezing Temperature, Alcohol Addition, and Molecular Weight. J. Appl. Polym. Sci. 2001, 81, 3008-3021. [CrossRef]

20. Wang, W.; Liu, K.; Dong, H.; Liu, W. High-performance liquid chromatography spectrometric analysis of tripterin in rat plasma. J. Chromatogr. Anal. Technol. Biomed. Life Sci. 2008, 863, 163-166. [CrossRef]

21. Food and Drug Administration. Guidance for Industry: Bioanalytical Method Validation; Food and Drug Administration: Rockville, MD, USA, 2018.

22. Gunasekaran, T.; Haile, T.; Nigusse, T.; Dhanaraju, M.D. Nanotechnology: an effective tool for enhancing bioavailability and bioactivity of phytomedicine. Asian Pac. J. Trop. Biomed. 2014, 4, S1-S7. [CrossRef] [PubMed]

23. Moss, D.M.; Siccardi, M. Optimizing nanomedicine pharmacokinetics using physiologically based pharmacokinetics modelling. Br. J. Pharmacol. 2014, 171, 3963-3979. [CrossRef] [PubMed]

24. Li, W.; Zhang, T.; Ye, Y.; Zhang, X.; Wu, B. Enhanced bioavailability of tripterine through lipid nanoparticles using broccoli-derived lipids as a carrier material. Int. J. Pharm. 2015, 495, 948-955. [CrossRef] [PubMed]

25. Yin, J.; Wang, P.; Yin, Y.; Hou, Y.; Song, X. Optimization on biodistribution and antitumor activity of tripterine using polymeric nanoparticles through RES saturation. Drug Deliv. 2017, 24, 1891-1897. [CrossRef] [PubMed]

26. Qi, X.; Qin, J.; Ma, N.; Chou, X.; Wu, Z. Solid self-microemulsifying dispersible tablets of celastrol: Formulation development, charaterization and bioavailability evaluation. Int. J. Pharm. 2014, 472, 40-47. [CrossRef]

27. Song, J.; Shi, F.; Zhang, Z.; Zhu, F.; Xue, J.; Tan, X.; Zhang, L.; Jia, X. Formulation and evaluation of celastrol-loaded liposomes. Molecules 2011, 16, 7880-7892. [CrossRef] [PubMed]

28. Rockwood, D.N.; Preda, R.C.; Yucel, T.; Wang, X.; Lovett, M.L.; Kaplan, D.L. Materials fabrication from Bombyx mori silk fibroin. Nat. Protoc. 2011, 12, 1612-1631. [CrossRef] 
29. Kundu, J.; Chung, Y.-I.; Kim, Y.H.; Tae, G.; Kundu, S.C. Silk fibroin nanoparticles for cellular uptake and control release. Int. J. Pharm. 2010, 388, 242-250. [CrossRef]

30. Papaseit, E.; Pérez-Mañá, C.; Mateus, J.A.; Pujadas, M.; Fonseca, F.; Torrens, M.; Olesti, E.; de la Torre, R.; Farré, M. Human Pharmacology of Mephedrone in Comparison with MDMA. Neuropsychopharmacology 2016, 41, 2704-2713. [CrossRef]

Sample Availability: Samples of the compounds celastrol pure drug and celastrol-loaded silk fibroin nanoparticles are available from the authors.

(C) 2019 by the authors. Licensee MDPI, Basel, Switzerland. This article is an open access article distributed under the terms and conditions of the Creative Commons Attribution (CC BY) license (http://creativecommons.org/licenses/by/4.0/). 